\title{
Software Package Evaluation for Lyapunov Exponent and Others Features of Signals Evaluating Condition Monitoring Performance of Nonlinear Dynamic Systems
}

\author{
Julio César Gomez-Mancilla ${ }^{1}$, Luis Manuel Palacios-Pineda ${ }^{2}$ and Valeriy Nosov ${ }^{1}$ \\ 1. Vibrations \& Rotordynamics Laboratory, ESIME (Superior School of Mechanical and Electrical Engineering), IPN (National \\ Polytechnic Institute), Zacatenco Professional Center, México, D. F. 07738, México \\ 2. Graduated and Research Department, Technological Institute of Pachuca, Pachuca 42083, México
}

Received: January 08, 2015 / Accepted: March 02, 2015 / Published: May 31, 2015.

\begin{abstract}
Efficient use of industrial equipment, increase its availability, safety and economic issues spur strong research on maintenance programs based on their operating conditions. Machines normally operate in a linear range, but when malfunctions occur, nonlinear behavior might set in. By studying and comparing five nonlinear features, which listed in decreasing order by their damage detection capability are: LLE (largest Lyapunov exponent), embedded dimension, Kappa determinism, time delay and cross error values; i.e., LLE performs best. Using somewhat similar ideas from Chaos control, i.e., vary the "mass imbalance" forcing parameters, we aim to stabilize the Lorenz equation. Quite interestingly, for certain imbalance excitation values, the system is stabilized. The previous even when paradigmatically chaotic parameters for Lorenz system are used (plus our forcing terms). This quasi-control approach is validated studying signals obtained from the previously mentioned lab test. Finally, it is concluded that analyzing and comparing nonlinear features extracted from baseline vs. malfunction condition (test acquired), one might increase the efficiency and the performance of machine condition monitoring.
\end{abstract}

Key words: Modified Lorenz equation, largest Lyapunov exponent, nonlinear features, chaos control, test validation.

\section{Introduction}

Technology based on vibration condition monitoring is largely used due to the necessity to increase equipment availability, safety and economic issues. Machines by their nature are designed to display linear behavior during normal operation. When machines have reasonable bounded amplitude and linear behavior, most of the times are considered as "healthy" systems. Particularly signals obtained from such "healthy system" normally can be analyzed with traditional techniques as FFT (fast Fourier transform), STFT (short time Fourier transform), wavelet, etc. Nevertheless, machines are always exposed to variable

Corresponding author: Julio César Gomez-Mancilla, Ph.D., professor, research fields: structural and mechanical vibrations, design, rotor non-linear dynamics, chaos. E-mail: gomezmancilla@gmail.com. workloads, insufficient or lack of maintenance and others aspects leading to the possibility of developing some kind of malfunction (unbalance, misalignment, steam-whirl, cracks, etc.), as a result depending on the magnitude of such malfunction, the dynamic condition of the machine changes and might become a system with certain degree of nonlinearity.

If a vibration signal is analyzed by linear processing techniques, information revealing malfunction characteristics might get lost, thus the need to use nonlinear processing tools. As an example, a modified Lorenz equation, where we add an external force, is analyzed. Nonlinear tools implemented in the Perc package [1] such as time delay, embedding dimension, error, determinism, stationarity and LLE (largest Lyapunov exponent), also time series are analyzed as explained by Ref. [2], and calculi applied to lab test. 
Studies in this area are good but few and seminal book by Randall [3] helps. Works by Wang, et al. [4, 5] use the pseudo-phase portrait to find the importance of using an adequate time delay $\tau$ value to create such portrait (in Ref. [6], Gomez-Mancilla also emphasizes $\tau$ proper estimation), sensitivity to detect faults in machinery is also studied. They demonstrated that, these nonlinear features can be effective parameters for fault condition prognosis. In Ref. [7], Antoine compares the Lyapunov exponent and Jacobian feature vector calculated from an experimental setup.

Similar to previous works by Gomez-Mancilla $[8,9]$ our aim is to discriminate and select nonlinear features from time signals acquired at normal baseline operation; while extracting features to characterize changes in the system dynamic behavior to compare vs. signal features when the machine has malfunctions.

\section{Lorenz Equation, Original and Modified}

In 1963, meteorologist Ed Lorenz derived a three dimensional relatively simple set of first-order nonlinear differential equations. He truncated the partial differential equations describing thermal driving of convection in the lower atmosphere (Eq. (1)):

$$
\begin{aligned}
& \dot{x}=\sigma(z-x) \\
& \dot{y}=x y+\rho x-y \\
& \dot{z}=x y-\beta z
\end{aligned}
$$

For certain parameters values, $\sigma, \rho, \beta$, i.e., $\sigma=10, \rho$ $=25, \beta=8 / 3$, the system displays an irregular $=25, \beta$
$=8 / 3$, the system displays an irregular deterministic chaotic behavior which may be characterized by the LLE. To study faulty systems, here sinusoidal terms are added aiming at simulate an external exciting rotating force. Such modified Lorenz equation is shown in Eq. (2):

$$
\begin{aligned}
& \dot{x}=\sigma(z-x)-F_{0} \sin (\omega t) \\
& \dot{y}=x y+\rho x-y+F_{0} \cos (\omega t) \\
& \dot{z}=x y-\beta z+F_{0} \sin (\omega t)
\end{aligned}
$$

Modifying Lorenz equation is some kind of chaos control as described by Refs. [10,11], yet we are not exactly perturbing nor varying any equation parameter values. By adding forcing terms, similarly as mass imbalance influences a rotating machine system, we explore and study the resulting system stability.

Using Matlab ODE45 (ordinary differential equation solver45) function, Eqs. (1) and (2) are solved with sample time equal to $0.01 \mathrm{~s}$ (4,000 data samples). Fig. 1 shows time series corresponding to $x(t), y(t)$ and $z(t)$ modified Lorenz system responses which are used to calculate nonlinear features; and for some parameter values Eqs. (1) and (2) might become chaotic. To calculate their features, nonlinear analysis package in Ref. [1] is used; data pre-processing is advised as Ref. [6] exposes. In accordance to Takens theorem [12], dynamic features from numerically simulated or acquired stationary and deterministic processes are extracted.
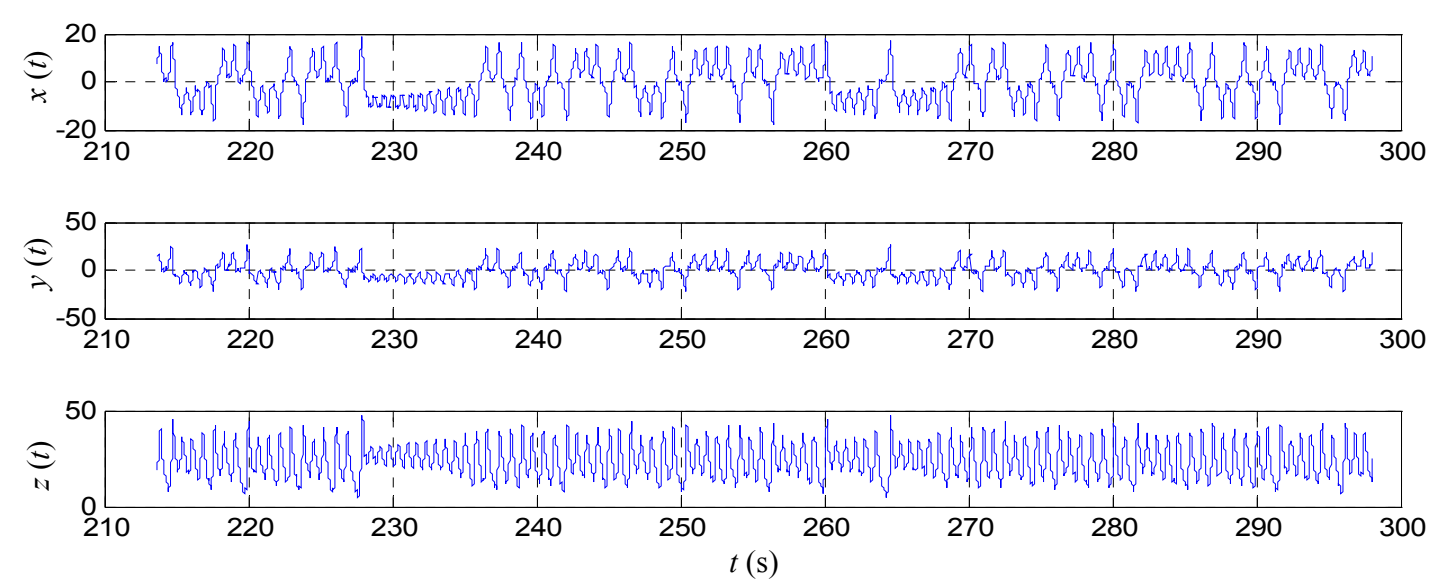

Fig. 1 Time responses $x(t), y(t), z(t)$ from Eq. (2) for $\sigma=10, \rho=28, \beta=8 / 3, F=50, \omega=30$, two competing attractors co-exist. 


\section{Nonlinear Time Series Analysis}

Nowadays, condition monitoring by different signal processing methods (frequency and time domain analysis, wavelet, etc.), can be realized. Yet, potential irregular nonlinear behavior arising from presence of malfunctions in actual machines motivates the use of powerful tools from nonlinear analysis. Following steps aim at calculating the LLE [1]. First, obtain a proper $\tau$ and embedding dimension $m$ values by mutual information [13] and false nearest neighbor methods [14], respectively. Next, ensure input data requirements are satisfied by testing if the system is deterministic [15] and stationary [16]. Then, using a single time series should verify if the reconstructed phase or embedded space seems congruent. Finally, using Wolf algorithm [17, 18], calculate the LLE and determine if the system is chaotic, or not.

\subsection{Embedded Space Reconstruction}

The reconstruction generates information about the unobserved (not measured) data, which allows predicting the rest of the state variables. Mathematical description is based on Takens' theorem [12], where using a single scalar time series $x(t)$ can be enough to reconstruct the state space. The previous since the coordinates are related to each other through a time delay $(\tau)[6,13]$. If $\tau$ is very small, then coordinates $x_{t}$ and $x_{(t+(m-1) \tau)}$ are numerically so close to each other, that can not be distinguished from each other. On the contrary, if $\tau$ is too large, then $x_{t}$ and $x_{(t+(m-1) \tau)}$ become independent of each other, in a statical sense.

Having properly determined $\tau$, the information obtained about other coordinates is large enough to allow us to introduce values at times $(t+\tau, t+2 \tau, \ldots$, $(t+(m-1) \tau)$ to substitute for the original coordinates. An embedding space having same/similar features as the original system, is therefore recreated (Eq. (3)).

$$
p(t)=\left(x_{t}, x_{t+\tau}, x_{t+2 \tau}, \ldots, x_{t+(m-1) \tau}\right)
$$

While, inspecting Fig. 2, the necessity for proper input parameters $\tau$ and $m$ becomes clear. Notice the quite different generated spaces.

\subsection{Some Nonlinear Features}

Time delay $(\tau)$ : to calculate the time delay, the MI (mutual information) method allows satisfactory results, better as compared to other methods (i.e., linear autocorrelation which are not studied in this work). From state $x_{t}+\tau$, equation for mutual information is:

$$
I(\tau)=-\sum_{h=1}^{j} \sum_{k=1}^{j} P_{h, k}\left(x_{t}, x_{t+\tau}\right) \ln \left[\frac{P_{h, k}\left(x_{t}, x_{t+\tau}\right)}{P_{h}\left(x_{t}\right) P_{k}\left(x_{t+\tau}\right)}\right]
$$

where, $P\left(x_{t}\right)$ and $P\left(x_{t}+\tau\right)$ are the probabilities that a variable assumes a certain value inside the $h$-th and the $k$-th bins, respectively. $P\left(x_{t}, x_{t}+{ }_{\tau}\right)$ is the joint probability that $x_{t}$ is in bin $h$ and $x_{t+\tau}$ is in bin $k$. If variables $x_{t}$ and $x_{t+\tau}$ are completely independent, it means that, these variables are not correlated, and $I(\tau)$ $=0$. Program input to mutual.exe are the number of bins $j$ and the expected maximal embedding delay.

Embedding dimension $(\mathrm{m})$ : mainly to provide a Euclidean space with a dimension large enough such that, the system dynamics can be unfolded without ambiguity. FNN (false nearest neighbor) method is an

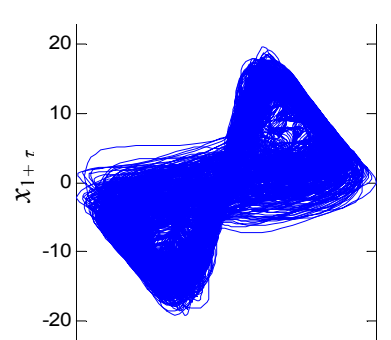

(a)

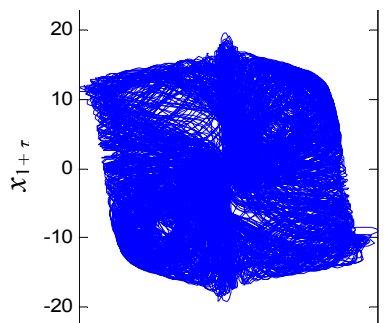

(b)

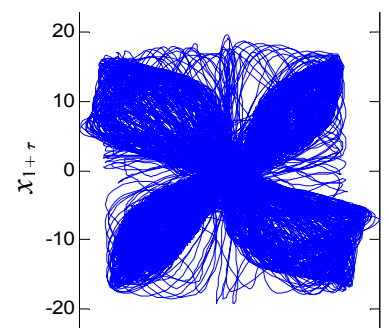

(c)

Fig. 2 State space reconstruction for the modified Lorenz system (Eq. (2)): $\sigma=10, \beta=8 / 3, \omega=30, F=50, m=3 ; \tau$ applied to generating signal $x(t)$ are: (a) $\tau=18$; (b) $\tau=58$; (c) $\tau=80$. 
efficient algorithm that determines the minimum embedding dimension $(m)$, see for instance [14]. The number of neighbors change along the signal path:

$$
r_{D}^{2}=\sum_{k=0}^{m-1}\left[x_{n+k t}-x_{n+k t}^{r}\right]^{2}
$$

Adding the proper time delay, transforms $m$ to $m+1$, creating a new coordinate system, if the distances change from one to another dimension, they are called false neighbors. Previous method is implemented and input to the fnn.exe code are $\tau$, and the minimal and maximal embedding dimension bound for which the FNN is to be determined.

A deterministic system occurs when through a set of ordinary differential equations, an initial state can determine its future state. Uniqueness of solutions is the property on which determinism test is built up. Kaplan and Glass [15] proposed that determinism test is required (where optimal $k$ is equal to 1 ). The vector field approximation for $V_{k}$ in the $k$-th box of the phase space is the average vector of all passes obtained according to Eq. (6), $P_{k}$ is the number of all passes through the $k$-th box. The code determinims.exe calculates a unique determinism parameter, inputs are time delay and number of data points.

$$
V_{K}=\frac{1}{P_{K}} \sum_{i=1}^{P_{K}} e_{i}
$$

The code stationarity.exe indicates if the system properties are kept constant during the data acquisition process. Kantz and Schrieber [2] propose to compare properties in one time series segment to another segment acting as a data base, and call it cross-prediction error, where, $\mathrm{x}_{t+\Delta t}$ is the based value, $\tilde{x}_{t+\Delta t}$ is the predicted value and $N$ is the number of trials made. Error should be minimal for a stationary system since $x_{t}$ and a neighbor pertain to the same data segment, this is implemented into the stationarity.exe code.

$$
\gamma=\sqrt{\frac{1}{N} \sum_{k=1}^{N}\left(\tilde{x}_{t+\Delta t}-x_{t+\Delta t}\right)^{2}}
$$

\subsection{LLE}

Lyapunov exponents deserve a special place, considering the divergence or convergence of nearby orbits they determine the system dependence on its initial conditions. An $m$ dimensional system has $m$ possible Lyapunov exponents; existence of positive LLE defines its unstable directions. The lyapmax.exe code implements Wolf, et al. algorithm [17]; input parameters are $\tau$ and $m$, then the LLE is calculated from:

$$
\lambda_{\infty}=\frac{1}{T} \log \frac{\|\delta x(t+T)\|}{\|\delta x(t)\|}
$$

Applying Wolf theory, the implicit numeric LLE calculation to each of the three (Eq. (2)) post-processed response results are compared. Based on the system Jacobian $J$, implicit numeric calculation estimates the perturbation exponential growth $\delta x$ :

$$
\frac{\mathrm{d} \delta x}{\mathrm{~d} t}=J(x) \delta x
$$

Fig. 3 shows how varying the "imbalance" and $\beta$ parameters induces significant changes in the system stability, in $\beta$ regions where the system is chaotic unstable; for instance and specifically, $F=50$ and $\omega=$ $3 \mathrm{r} / \mathrm{s}$, are capable to control the system and turn it stable. While, varying $\beta$ parameter, instability and stability are predicted by positive and negative LLE values, respectively. Except for the purple line analyzing $z(t)$ response, all LLE values are calculated by the Wolf-Jacobi algorithm numerically applied to Eq. (2); recall that this implicit procedure [1,17] does not require individual analysis for each system signal response. Using typical $\sigma=10, \rho=28$, range $0<\beta<5$, Fig. 3 considered three distinct cases:

(1) typical unperturbed Lorenz Eq. (1), red color line;

(2) imbalance forced Lorenz Eq. (2), $F=50, \omega=30 \mathrm{r} / \mathrm{s}$, calculated both ways: by Wolf-Jacobi algorithm shown by blue line, and also applying Perc package [1] to $z(t)$ shown by purple line;

(3) imbalance controlled Lorenz, Eq. (2), $F=50, \omega$ $=3 \mathrm{r} / \mathrm{s}$, green color line. Notice three new system stability $\beta$ regions, including at the dreadful value $\beta=$ $8 / 3$ where a stable limit cycle orbit occurs. 


\section{Application to Experimental Tests}

Used lab experimental equipment consists of a rotor system, a MMFS (modified machine fault simulator) [19], Bently Nevada WinADRE (windows automated diagnostics for rotating equipment), and LabVIEW-NI (National Instruments) data acquisition system. Fig. 4 shows most lab testing equipment.

Test under different conditions are performed: integral (undamaged) rotor, bent shaft at two levels and unbalance malfunctions, a damaged (cracked shaft) rotor. Analyzed malfunctions interact. Proximity sensors sample at $0.0002 \mathrm{~s}$. Typical signals at constant speed near 1st machine resonance at test conditions, undamaged and bent malfunction are shown in Fig. 5 .
Extracted nonlinear features for undamaged baseline operation are shown in Table 1 illustrating variation of the nonlinear features at different rotational speeds and up to the first resonance frequency. The embedding dimension is fixed at $m=4$ and remains constant for all the analyzed experiments. Notice that all test baseline conditions yield negative Lyapunov exponents (stable behavior).

Changes with respect to the corresponding baseline for the $j$ nonlinear feature, can be calculated as:

$$
\begin{aligned}
& \text { Percentage change }_{j}= \\
& \frac{\text { Feature value }_{j}-\text { Baseline value }_{\text {Baseline value }}}{\text { B }^{2}} \times 100 \%
\end{aligned}
$$

Integral shaft system malfunctions are bending and

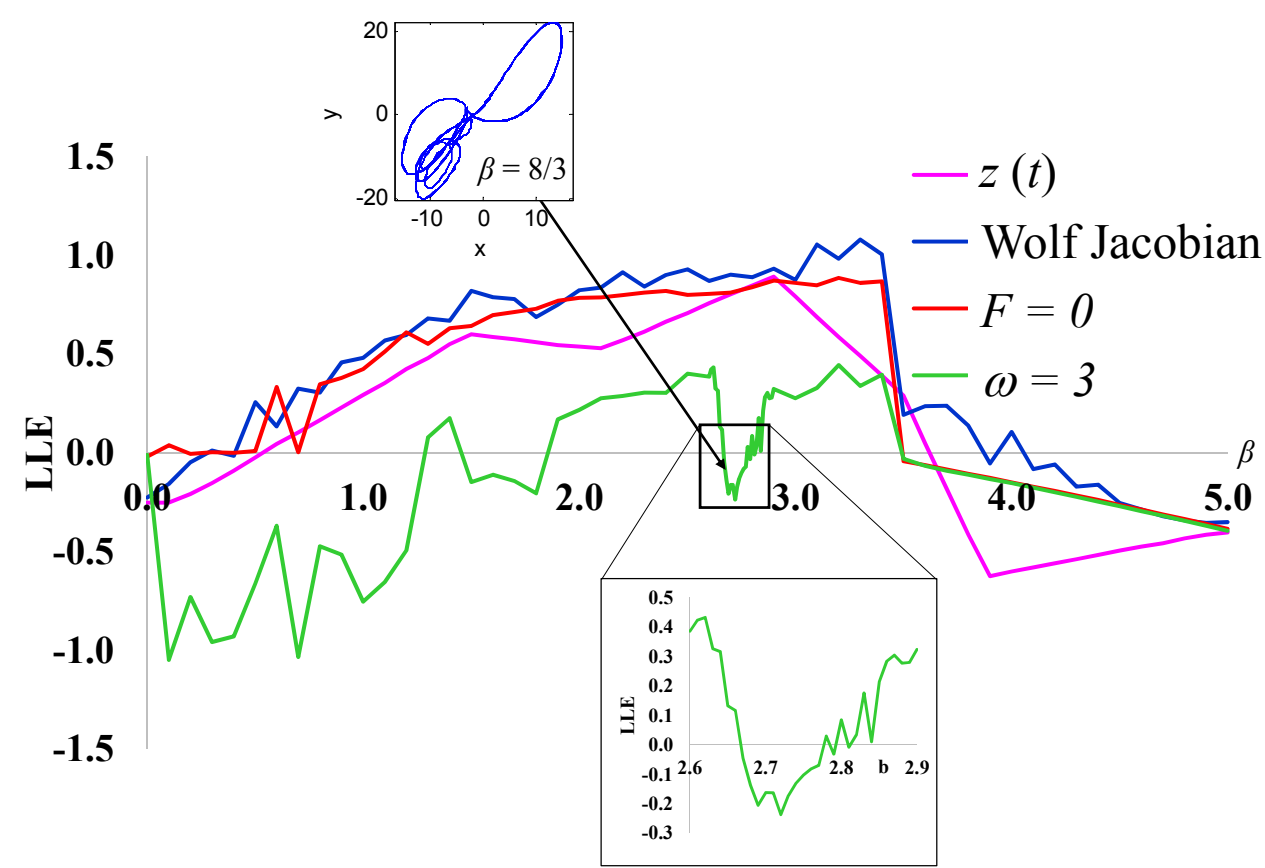

Fig. 3 LLE stability estimated by Wolf-Jacobi and $z(t)$ (Eq. (2)). For $\sigma=10 ; \rho=28 ; F=0$ and 50; $\omega=3$ and $30 ; 0<\beta<5$.

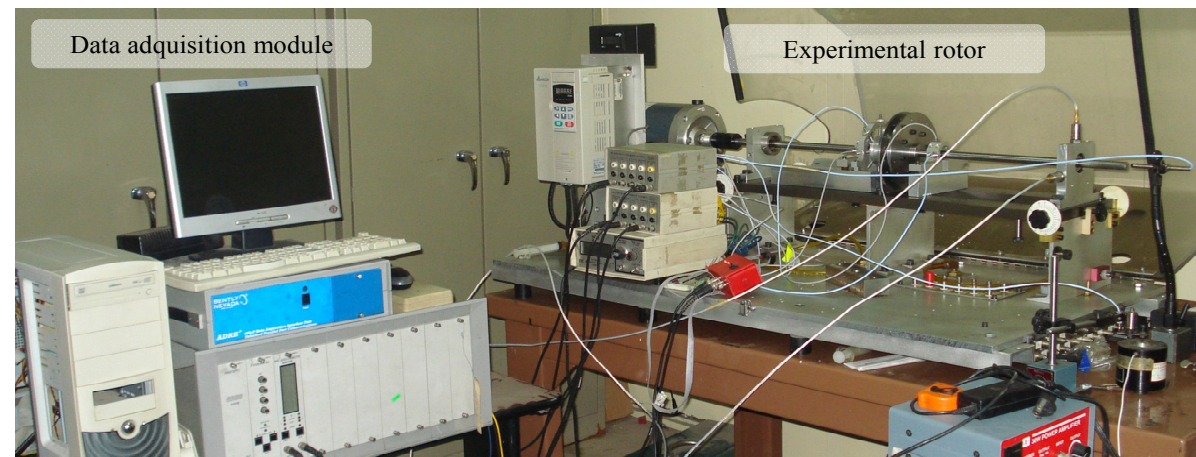

Fig. 4 Main lab test equipment. Instrumented MMFS rotor malfunctions and adapted to simulate a cracked shaft. 


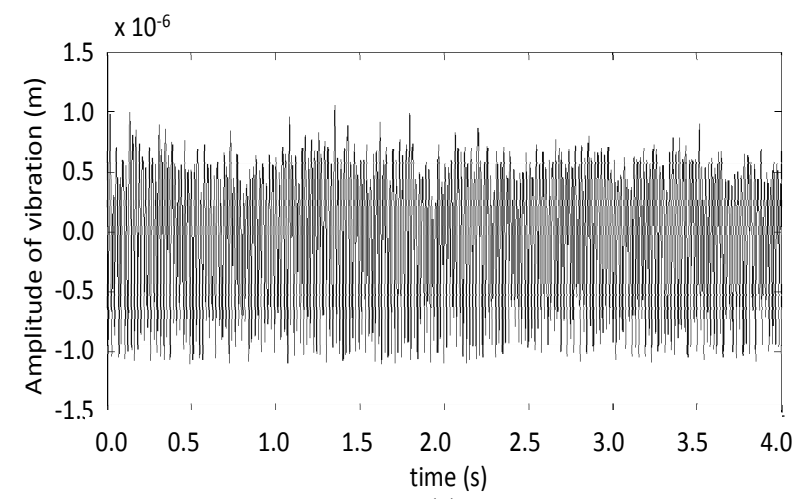

(a)

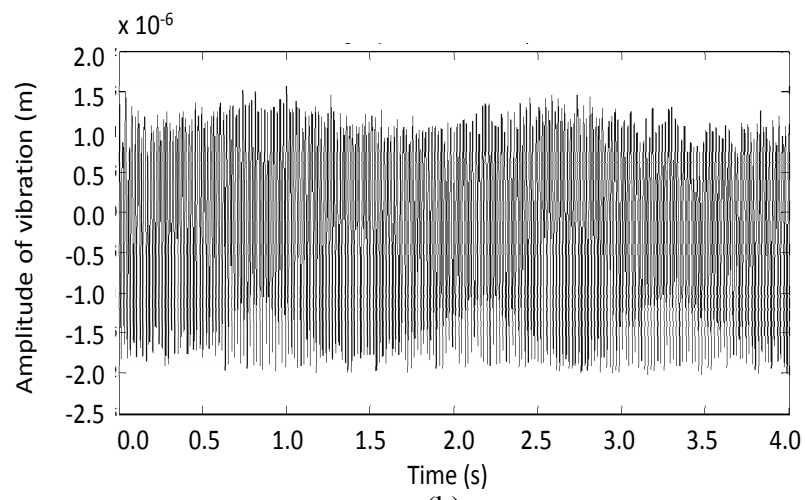

(b)

Fig. 5 Filtered proximitor signals from an MMFS integral system rotating near first resonance (normalized frequency $\omega / \omega_{n}$ = 0.89): (a) baseline integral system; (b) system with a malfunction (level 1 bending).

Table 1 Extrated nonlinear features at baseline operation, different normalized rotation speeds $\omega / \omega_{n}$.

\begin{tabular}{lllllllll}
\hline$\omega / \omega_{n}$ & 0.445 & 0.455 & 0.500 & 0.650 & 0.660 & 0.890 & 0.910 & 1.000 \\
\hline Time delay & 32 & 31 & 30 & 32 & 31 & 29 & 31 & 29 \\
Cross prediction error & 0.0332 & 0.0365 & 0.0395 & 0.0321 & 0.0397 & 0.0412 & 0.0385 & 0.0370 \\
Determinism & 0.865 & 0.843 & 0.895 & 0.885 & 0.843 & 0.938 & 0.895 & 0.920 \\
LLE & -0.943 & -0.958 & -0.693 & -0.780 & -0.808 & -0.595 & -0.690 & -0.490 \\
\hline
\end{tabular}

unbalance. They are classified as: bending levels 1,2 and 3, and only level 1 unbalance (unbalance mass equal to $0.5 \mathrm{~g}$ ). Two shafts damage are: crack depth level 1 means $10 \%$ crack depth normalized by the diameter; and crack depth level 2 is a $30 \%$ normalized crack depth. Malfunction combinations are analyzed.

Increase in malfunction and damaged levels are introduced into the MMFS test set up, signals are acquired, filtered using nonlinearnoisereduction.exe code and then numerically processed. Such non-conventional filtering carefully treats the signals as to not overly suppress nonlinear data which might contain relevant malfunction information. The de-noising procedure has a relevant positive effect on the routines input parameters and strongly impacts all the calculation results.

Nonlinear features performance: Five nonlinear features trends and patterns are evaluated by a series of lab test with known conditions. Table 2 shows extracted features including LLE, $\tau, m$, determinism and cross-prediction error values compiled from Figs. $6 \mathrm{a}$ and $6 \mathrm{~b}$ plots, yielding adequate values close to zero, 0.010 and 0.020 . Previous signal extracted values insure fulfilment of the stationary requirement, which also are Lyapunov stable (i.e., LLE negative). On the other hand, although in Fig. 6c 2-D graph shows no local error minimization along the 45 degree angular orientation and a small average error of 0.040 , it has a high maximum error, 0.069. Moreover, positive LLE > 0 , clearly indicates a chaotic unstable machine behavior. Test signal for this cracked rotor case is shown in Fig. 7, where chaos can not be perceived by mere ball eye inspection. The other feature seemed less sensitive to malfunctions: determinism does not have sensitivity to detect malfunctions. While, different kinds of errors directly evaluating the stationarity dynamic characteristic might have certain small diagnosing potential.

In Fig. 8, left-right sense along the horizontal axis indicates a worsening of machine condition. In Fig. 8a, both embedded dimension and determinism show low sensibility to increasing worsening malfunction level, with the exception for a highly damaged machine which has stronger nonlinear dynamics and jumps from $m$ dimension 2 to 4 . While in Fig. $8 \mathrm{~b}$, a fluctuating yet slight increasing trend, can be perceived in the time delay feature, while, the average cross-predicted error values seem to fluctuate with no 
clear trend. Fig. 9 shows the LLE feature for the damaged system operating at 6 malfunction, including the worse level induced to the MMFS (i.e., bending level 3 and crack level 2). Notice a clear monotonically increase of the LLE feature, which is congruent with the machine worsening condition.

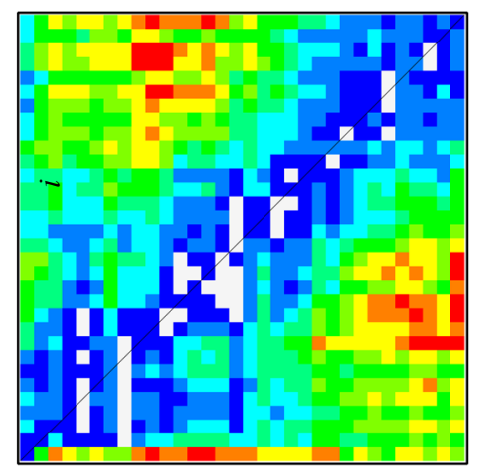

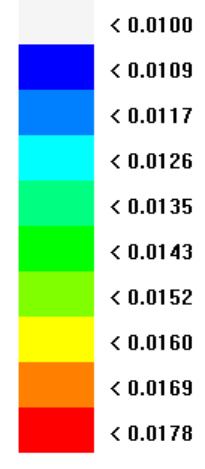

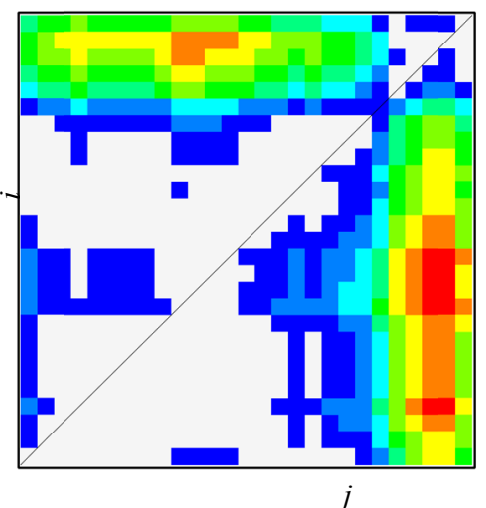

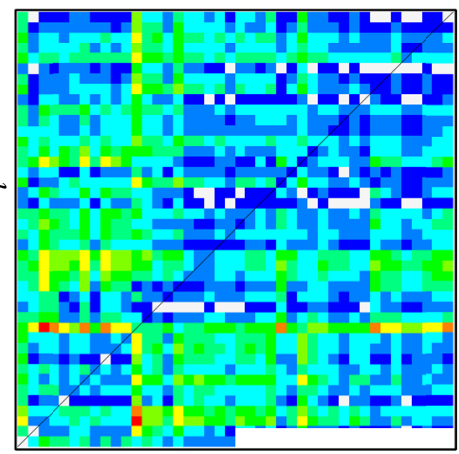

j

(c)
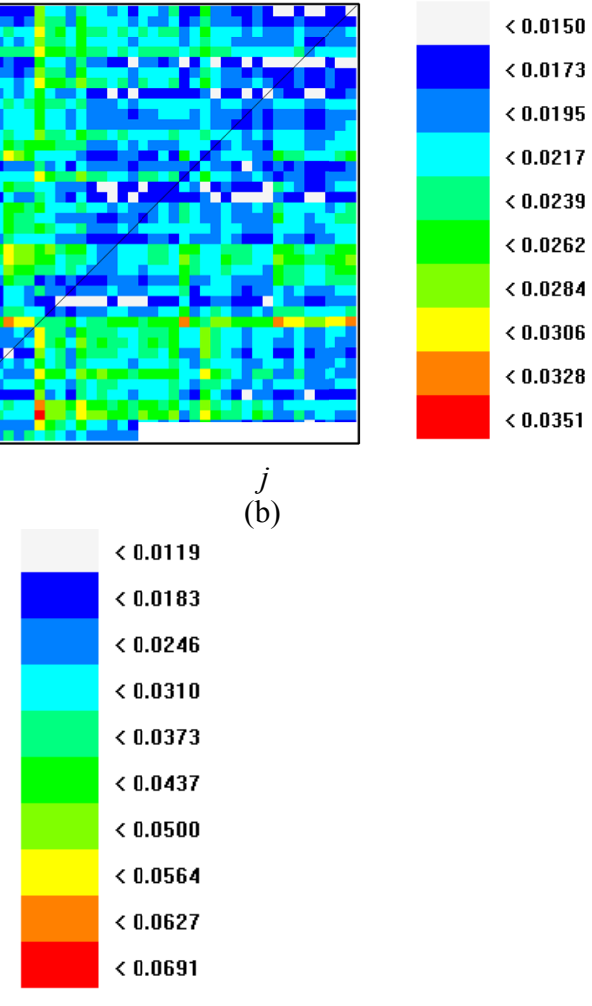

(b) 

Condition Monitoring Performance of Nonlinear Dynamic Systems

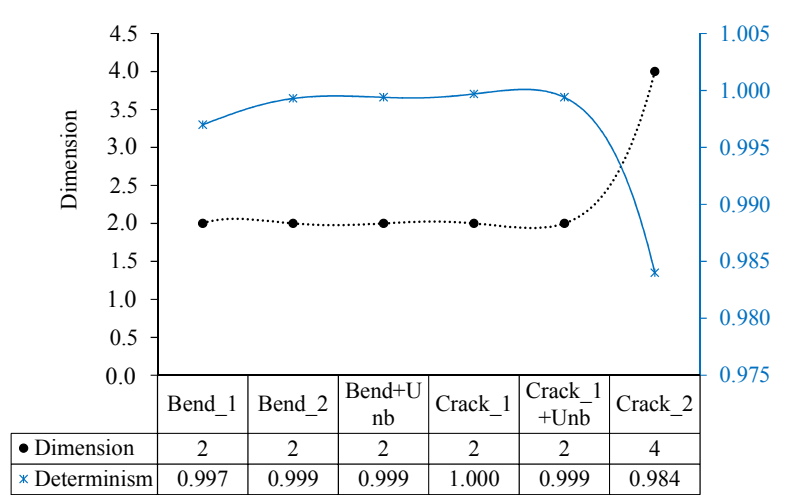

(a)

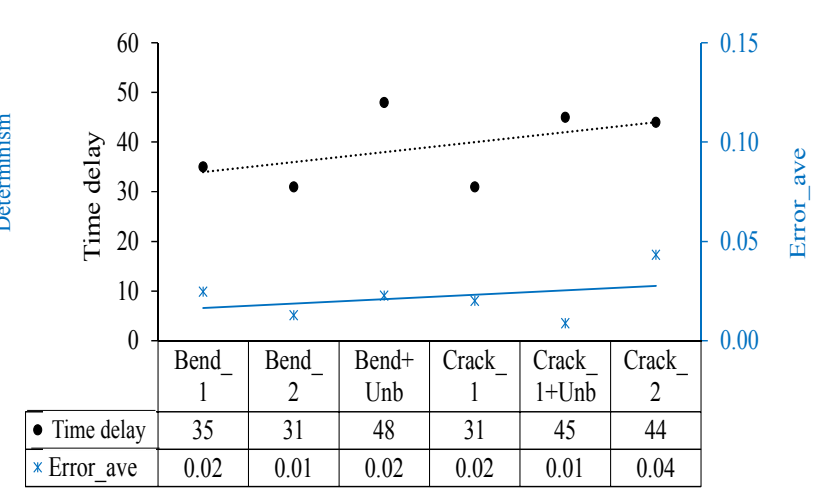

(b)

Fig. 8 Left-right sense along horizontal axis indicates worsening machine malfunction severity level: (a) embedded dimension and determinism features; (b) embedded time delay $\tau$ and average cross-predicted error.

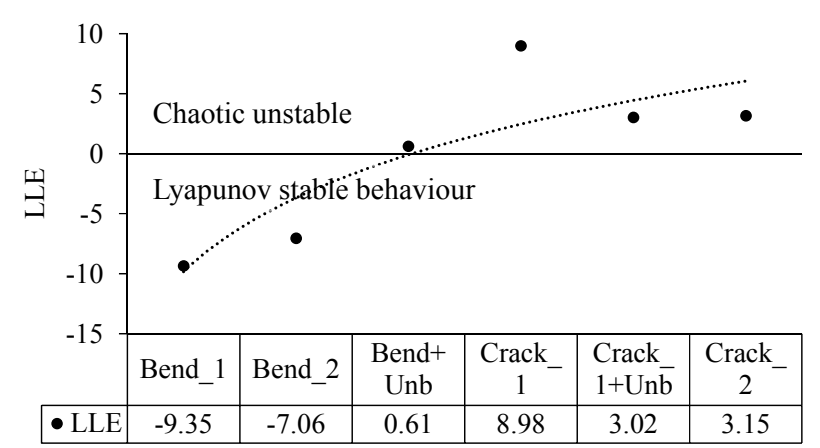

Fig. 9 The LLE feature shows a clear increasing trend, congruent with the machine malfunction severity level.

\section{Conclusions}

Increased availability of industrial equipment, safety and economic issues motivate research in maintenance programs based on monitored operating conditions. Machine behavior might turn nonlinear when operating with malfunctions. Typically linear excitation forcing terms are difficult to characterize, while nonlinear dynamics inducing large amplitude and/or chaotic behavior (i.e., certain deep breathing cracked shafts) are less difficult to identify. Five nonlinear features are extracted where the LLE displays higher sensitivity to machine malfunctions, since corresponding Lyapunov exponent feature tends to exhibit a decreasingly stability margin.

Moreover, to find potential reasons that would explain why most lab test signals obtained from malfunctioning equipment seemed dynamically sound, stability calculations extract nonlinear features from signals coming from actual machine test, and numerically, simulating an externally forced Lorenz system. Added forcing terms are similar to a rotating mass imbalance influence. Next using Chaos Control similar concepts, i.e., vary the "mass imbalance" forcing parameters we aim to stabilize such modified Lorenz equation. Again both Wolf algorithms: Jacobian and that implemented by Perc M. are used.

Quite interestingly for certain values of the imbalance excitation in Eq. (2), the system can be stabilized, the previous even when paradigmatically chaotic parameters for Lorenz system are used, see Fig. 3. This quasi control approach is validated studying signals obtained from previously mentioned lab test (Fig. 9), where residual mass imbalance is omnipresent in the test.

Finally, it is concluded that, analyzing and comparing baseline vs. malfunction extracted nonlinear features (acquired from equipment) can increase the performance of machine condition monitoring.

\section{Acknowledgments}

Authors acknowledge the CONACYT (National Council for Science and Technology), IPN (National Polytechnic Institute) and TecNM (National Technologic of Mexico) for sabbatical year at Trieste University, Italy for prof. Gomez and for scholarships received.

\section{References}

[1] Perc, M. 2006. "Introducing Nonlinear Time Series Analysis in Undergraduate Courses." Fizika A (Zagreb) 15 (2): 91-112. 

Condition Monitoring Performance of Nonlinear Dynamic Systems

[2] Kantz, H., and Schrieber, T. 2004. Nonlinear Time Series Analysis. Cambridge: Cambridge University Press.

[3] Randall, R. 2011. Vibration-Based Condition Monitoring. New Delhi: Wiley.

[4] Wang, B., and Lin, R. 2003. "The Application of Pseudo-phase Portrait in Machine Condition Monitoring." Journal of Sound and Vibration 259 (1): 1-16.

[5] Wang, B., and Zhaohui, R. 2012. "Study on Fault Diagnosis of Rotating Machinery based on Lyapunov Dimension and Exponent Energy Spectrum." Advanced Materials Research 591-593 (November): 2042-5.

[6] Gomez-Mancilla, J. C., Huesca-Lazcano, E., Palacios-Pineda, L., and M'Sirdi, N. 2015. "Signal Treatment Method to Properly Extract the Time Delay Feature to Characterize Nonlinear Systems." MGEF (Mediterranean Green Energy Forum), Marrakech, Morocco.

[7] Antoine, C. 2011. "An Alternative to Lyapunov Exponent as Damage Sensitive Feature." Smart Materials and Structures 20 (2): 025017.

[8] Gómez-Mancilla, J. C., Sinou, J. J., Nosov, V. R., Thouverez, F., and Zambrano, A. 2004. "The influence of Crack-Imbalance Orientation and Orbital Evolution for an Extended Cracked Jeffcott Rotor." Comptes Rendus Mecanique 12 (332): 955-62.

[9] Machorro-Lopez, J. M., Adams, D. E., Gómez-Mancilla, J. C., and Gul, K. 2009. "Identification of Damaged Shafts Using Active-Sensing Simulation and Experimentation." $J$. of Sound and Vibration 327 (3-5): 368-90.

[10] Heitor, F., Pereira-Pinto, I., and Ferreira, A. M. 2005. "State Space Reconstruction Using Extended State
Observers to Control Chaos in a Nonlinear Pendulum." International Journal of Bifurcation and Chaos 15 (12): 4051-63.

[11] Eckehard, S., and Heinz, G. S. 2007. Handbook of Chaos Control. Weinheim: Wiley-VCH.

[12] Takens, F. 1981. "Detecting Strange Attractor in Turbulence.” In Lecture Notes in Mathematics, edited by Rand, D. A., and Young, L. S. Vol. 898. Berlin: Springer-Verlag, 366-81.

[13] Andrew, M. F., and Swinney, H. L. 1986. "Independent Coordinates for Strange Attractors from Mutual Information." Physical Review 33 (2): 1134-40.

[14] Kennel, M. B., Brown, R., and Abarbanel, H. D. 1992. "Determining Embedding Dimension for Phase Space Reconstruction Using a Geometrical Construction." Phys. Rev. A 45 (6): 3403-11.

[15] Kaplan, D. T., and Glass, L. 1992. "Direct Test for Deter-minism in a Time Series." Phys. Rev. Lett. 68 (4): 427-30.

[16] Abarbanel, H. D. 1996. Analysis of Observed Chaotic Data. New York, NY: Springer-Verlag.

[17] Wolf, A., Swift, J. B., Swinney, H. L., and Vastano, J. A. 1985. "Determining Lyapunov Exponents from a Time Series." Physica D 16 (3): 285-317.

[18] Shaw, R. 1981. Strange Attractors, Chaotic Behavior and Information Flow. Z. Naturforsch. A: CiteSeer.

[19] SpectraQuest, 2105. "MFS (Machinery Fault Simulator)". SpectraQuest. Accessed December 12, 2014. http://spectraquest.com/products/simulators/machinery-fa ult-simulators. 